\title{
Software Engineering and Swarm-Based Systems
}

\author{
Michael G. Hinchey \\ NASA GFSC \\ Greenbelt, MD, USA \\ michael.g.hinchey@nasa.gov
}

\author{
Roy Sterritt \\ University of Ulster \\ Northern Ireland \\ r.sterritt@ulster.ac.uk
}

\author{
Joaquín Peña \\ University of Seville \\ Seville, Spain \\ joaquinp@us.es
}

\author{
Christopher A. Rouff \\ SAIC, ACBU \\ McLean, VA, USA \\ rouffc@saic.com
}

\begin{abstract}
We discuss two software engineering aspects in the development of complex swarm-based systems.

\section{Categories and Subject Descriptors}

D.2.2 [Software Engineering]: Requirements/Specifications languages, methodologies, tools.

General Terms

Reliability, Languages, Verification.

Keywords

Autonomic Computing, Formal Methods, Multiagent Systems, Swarms.

\section{INTRODUCTION}

NASA researchers have been investigating various possible concept missions that would greatly advance future space exploration capabilities. The concept mission that we have focused on exploits the principles of autonomic computing as well as being based on the use of intelligent swarms, whereby a (potentially large) number of similar spacecraft collaborate to achieve mission goals. The intent is that such systems not only can be sent to explore remote and harsh environments but also are endowed with greater degrees of protection and longevity to achieve mission goals [1].
\end{abstract}

\section{ANTS}

ANTS (Autonomous Nano Technology Swarm) is a concept mission based on swarms. Its PAM (Prospecting Asteroid Mission) sub-mission involves the collection of scientific data from the asteroid belt. 1000 spacecraft each carrying a single instrument are launched from a Lagrangian point and form subswarms to identify asteroids of interest. Interested readers are directed to the ANTS website (http://ants.gsfc.nasa.gov/), or to [2], for further details.

\section{SOFTWARE ENGINEERING}

\subsection{Formal Methods}

The FAST (Formal Approaches to Swarm Technologies) project has been looking at the issues related to formally specifying and verifying swarm-based systems as exemplified by ANTS, and uses the PAM sub-mission for its investigation. The project surveyed a wide number of formal approaches, ranking them on their underlying formality, tool support, expressiveness, support for concurrency, support for expression of goals, prior use in agentbased and swarm-like applications, etc. The result is that the project came to the (not unexpected) conclusion that no single

This paper is authored by an employee(s) of the United States Government and is in the public domain.

ICSE SEAMS'06, May 21-22, 2006, Shanghai, China. Copyright 2006 ACM 1-59593-085-X/06/0005 ...\$5.00. formal notation would be sufficient for such systems and a hybrid method would be required. The project has been working on developing such a hybrid method and support tools are planned. A detailed account of the FAST project is given in [3]

\subsection{Model-Driven Architecture Approach for Policy Deployment}

The development of any autonomic system will require that various policies are determined for the correct operation of the system. These policies are used for guiding the development of a self-managing system, and can be considered to be very high level requirements, and must be implemented by the autonomic managers, etc. at run time.

We are investigating an MDA approach, based on applying policies to swarms, avoiding platform-dependent details, and using MDA transformations of models through implementation. This is based on our previous work applying an Agent-Oriented methodology called MaCMAS (Methodology Fragment for Analyzing Complex Multi-Agent Systems) to modeling, specifying and deploying policies at runtime. We are working on automating the process of adding new policies, essentially using two UML-based Platform Independent Models (PIMS) for specifying autonomous and autonomic properties of the system and an operation to transform these models in order to implement the changes specified by the policy into the running system [4].

\section{REFERENCES}

[1] M.G. Hinchey, J.L. Rash, W.F. Truszkowski, C.A. Rouff \& R. Sterritt "You Can't Get There From Here! Problems and some potential solutions in developing new classes of complex systems", Journal of Integrated Design And Process Science, Vol. 9, No. 1, IOS Press, Pages 1-16

[2] M.G. Hinchey, J.L. Rash, W.F. Truszkowski, C.A. Rouff, R. Sterritt, "Challenges of Developing New Classes of NASA Self-Managing Missions", In Proc. IEEE International conference on Parallel and Distributed Systems (Workshops), pp 463-467.

[3] C.A. Rouff, M.G. Hinchey, W.F. Truszkowski, J.L. Rash, "Experiences Applying Formal Approaches in the Development of Swarm-Based Space Exploration Systems", International Journal of Sofware Tools for Technology Transfer, 2006, to appear.

[4] J. Peña, M.G.Hinchey, R. Sterritt, "Towards Modeling, Specifying and Deploying Policies in Autonomous and Autonomic Systems using an AOSE Methodology", In Proc. $3^{r d}$ IEEE Workshop on Engineering Autonomic Systems (EASe 2006), Potsdam, Germany, March 2006. 